\title{
Kernos
}

Revue internationale et pluridisciplinaire de religion grecque antique

3 | 1990

Varia

\section{Au carrefour de la Mégaride. Devins et oracles}

\section{Pierre Lévêque et Claudia Antonetti}

Édition électronique
URL : http://journals.openedition.org/kernos/988

DOI : 10.4000/kernos.988

ISSN : 2034-7871

\section{Éditeur}

Centre international d'étude de la religion grecque antique

\section{Édition imprimée}

Date de publication : 1 janvier 1990

ISSN : 0776-3824

\section{Référence électronique}

Pierre Lévêque et Claudia Antonetti, «Au carrefour de la Mégaride. Devins et oracles », Kernos [En ligne], 3 | 1990, mis en ligne le 19 avril 2011, consulté le 01 mai 2019. URL : http:// journals.openedition.org/kernos/988; DOI : 10.4000/kernos.988 


\section{AU CARREFOUR DE LA MÉGARIDE. DEVINS ET ORACLES}

Point de rencontre, et d'arrimage, de différentes traditions politiques et culturelles au carrefour des routes pour le Péloponnèse, la Mégaride - pays de parcours par excellence - s'est constituée en État par un synœcisme de la fin du VIIIe siècle ${ }^{1}$ et s'est créé une tradition nationale propre, quoiqu'éclectique. Dans cette ambiance complexe, elle présente souvent, au plan religieux et mythique, la version mineure de traditions ailleurs bien plus développées ${ }^{2}$. Cependant ces «doublets» ne sont pas sans intérêt et, considérés dans leur ensemble, ils relèvent d'une certaine logique, qui plonge souvent dans la plus haute antiquité. Ce qui frappe le plus quand on étudie la documentation de la Mégaride, c'est l'importance des devins appartenant aux grandes familles de manteis : Calchas et Mélampous. C'est aussi la rareté des oracles, puisqu'est seul attesté l'épimanteion de Nuit sur l'acropole orientale de la cité ${ }^{3}$.

\section{Calchas}

Calchas est la première des figures mantiques que la tradition localise à Mégare. Selon Pausanias, Agamemnon vint l'y chercher, avant l'expédition de Troie; pendant ce séjour, l'Atride fonda un temple d'Artémis. À Mégare, il y avait aussi un hérôon d'Iphigénie, morte dans la ville (Paus., I, 43, 1). Les critiques ont vu, dans ce récit, la prétention de Mégare à s'insérer dans l'épos homérique, d'où elle est exclue ${ }^{4}$, ou une polémique anti-athénienne, visant à priver de

1 Sur la tradition de la dorisation de la Mégaride, cf. L. PICCIRILLI, Megarika (Testimonianze e frammenti), Pisa, 1975, fr. 2a, 2b (Dieuchidas) ; 2b, 19 (Hoi Megareis) ; R.P. LEGON, Megara (The political History of a Greek city-state to 336 B.C.), Ithaca - London, 1981, p. 41-58. Sur le syncecisme, cf. M. MoGGI, $I$ sinecismi interstatali greci, I (dalle origini al 338 a. C.), Pisa, 1976, p. 29-34.

2 Sur la religion mégarienne, cf. encore K. HANELl, Megarische Studien, Lund, 1934, p. 92-109, 161-190 et passim. Pour les données des sources, cf. E. MEYER, Megara, in RE, XV, 1 (1931), col. 201-203 et Suppl. XII (1970), col. 850-851.

3 Nous laissons de côté l'oracle d'Héra Acraia à Pérachora, qui appartint un temps à Mégare jusqu'à son appropriation par Corinthe en 750-700. Pour un exposé des différentes positions, cf. LEGON, p. 54 sq., 69 sq., 77 sq.

4 F. JACOBY, Theognis, SPAW, 1931, p. 100. Sur Mégare et le Catalogue des vaisseaux, cf., pour une version locale, STRAB., IX, 1, 10 (C394). 
fondement la légende locale de Brauron 5 . Pausanias même, dans le contexte où il expose la variante mégarienne du mythe d'Iphigénie, rappelle les autres versions qu'il connaît, l'arcadienne, celle du Catalogue des femmes et celle d'Hérodote (ibid.).

En outre, on remarque que, dans la généalogie royale de Mégare, le dernier roi, qui perd son pouvoir et est tué pour son hybris, est Hypérion, fils d'Agamemnon ${ }^{6}$ (Paus., I, 43, 3).

Pour étonnante qu'elle soit, il convient toutefois de prêter une certaine attention à l'histoire mégarienne, que d'autres éléments pourraient corroborer ${ }^{7}$.

Pour le sanctuaire d'Artémis, dédié par Agamemnon, il faut souligner que Théognis met en rapport direct le sacrifice de l'héroïne avec le culte de cette déesse (v. 11). En outre, des études récentes sur l'iconographie de l'Artémis Soteira à Mégare concluent en faveur de son identification avec la Phosphoros, hypostase de la déesse étroitement liée à la figure d'Hécate et au sacrifice d'Iphigénie ${ }^{8}$. De plus, dans les colonies de Mégare, les déesses assimilées à Artémis (Phosphoros, Parthénos, Chrysè) sont en relation avec Iphigénie et Hécate ${ }^{9}$. Il y a de bonnes raisons de croire que, des trois sanctuaires d'Artémis signalés par Pausanias à Mégare (I, 40,2;41,3;41,1), deux coïncident, à savoir le «vieux sanctuaire» de la Soteira, situé entre les deux acropoles, et celui qu'Agamemnon aurait fondé, tandis que le temple d'Artémis et Apollon Agraioi, consacré par Alcathoos, se trouve dans le secteur Nord de la ville ${ }^{10}$.

Si la création d'un temple en l'honneur d'Artémis s'explique par le retard de la flotte achéenne en partance pour Troie, il faut noter que cette flotte ne pouvait stationner, en Mégaride, qu'à Nisée, le port de Mégare sur le golfe Saronique. L'ensemble côtier de Nisée, avec la petite île de Minoa - au nom crétois si éloquent -, est le théâtre des légendes athéniennes et béotiennes sur l'invasion de la Mégaride par Minos, que les Mégariens contestaient ${ }^{11}$. Sur le plan mythique, c'était à des descendants de Poséidon qu'on attribuait la libération de l'hégémonie

5 PICCIRILLI, op. cit., p. 118-119, 160-161.

6 Cf. déjà HANELL, op. cit., 95-97.

7 Cf. aussi, dans ce sens, L. BESchI-D. Must, PAUSANIA, Guida della Grecia, I, L'Attica, Milano, 1982, p. 433.

8 A. Muller, Megarika VII, in BCH, 105, 1981, p. 222-225.

9 HANELL, op. cit., p. 95-97, 184-188.

10 MULLER, Megarika VII, p. 224-225.

11 PICCIRLLI, op. cit., p. 85-90. Cf. BESCHI-MUST, op. cit., p. 438-439. 
crétoise (dans la version béotienne, à Mégareus, fils de Poséidon $\mathrm{d}^{\prime}$ Onchestos) ${ }^{12}$. Se révèlent concordants le témoignage de Thucydide, qui cite les sanctuaires de Poséidon et d'Ényalios dans la zone du port mégarien (IV, 67,$2 ; 118,4$ ) et la notice de Pausanias, qui signale la présence d'un hiéron de Déméter Malophoros et du tombeau du roi Lélégos, fils de Poséidon, venu d'Égypte (I, 44, 3). L'ensemble religieux du «noyau mégarien côtier" reflète sans nul doute une strate homogène créto-mycénienne qui survit jusqu'à l'époque historique. L'archéologie confirme ce tableau: les seules trouvailles sûres d'installations mycéniennes proviennent de la zone de Nisée, tandis que le site de la ville de Mégare n'a encore rien fourni pour les hautes époques ${ }^{13}$. Le nom même de la cité est récent, datant de l'âge du Fer et correspondant peut-être à un programme de développement, s'il est vrai qu'il faut l'entendre comme «le grand hall» 14 .

Revenons à Calchas. L'une des caractéristiques de sa figure dans Homère (avant les développements ultérieurs) est celle d'un personnage étroitement lié à Agamemnon et au sacrifice d'Iphigénie en Aulide (Il., II, 300 sq.). Il est défini aussi bien comme le "meilleur des devins" (I, 69-72) que comme le «devin des malheurs» (I, 105-106). Il est censé recevoir son inspiration d'Apollon (I, 71, 75), mais, dans un épisode du XIIIe chant de l'Iliade, c'est Poséidon qui s'incarne en lui pour exhorter les Achéens à combattre devant leurs nefs. Poséidon gaièochos, ennosigaios, "maître de la terre" et "ébranleur de la terre», harangue les Argiens, «sorti de la mer profonde, ayant pénétré dans le corps de Calchas et dans sa voix infatigable» (XIII, 43-45). Il s'adresse aux deux Ajax; il les touche avec un bâton pour les rendre hardis et il s'envole ensuite, comme un épervier (ibid., 59-65). À ce mouvement les deux héros reconnaissent la présence divine, sous les atours du devin Calchas (ibid., 66-72).

Cet épisode, exceptionnel, montre une véritable possession du devin par Poséidon, et cela en connexion avec les deux Ajax, modèle de ces couples héroïques typiques de la phase la plus reculée de l'épos. L'un d'eux, le fils de Télamon, est le prototype même du guerrier mycénien

12 PICCIRILLI, op. cit., p. 83-84.

13 Cf. A. Muller, De Nisée à Mégare, dans Chronique d'une journée mégarienne, in $M E F R, 95$ (1983), 2, p. 619-623. Contra, mais sans analyse des données archéologiques, C. BÉRARD, Urbanisation à Mégara Nisaea et urbanisme à Mégara Hyblaea, ibid., p. 636-638.

14 Hypothèse notamment de K.J. RIGSBY, Megara and Tripodiscus, in GRBS, 28 (1987), 1, p. 99-101. 
ancien, lourdement armé ${ }^{15}$; il est le roi de Salamine et règne aussi sur la côte de la Mégaride ${ }^{16}$.

Dans ce tableau, tous les éléments sont cohérents: Calchas, Agamemnon, Ajax et le culte de Poséidon sur la côte mégarienne, et forment un ensemble mycénien, comme le montrent, d'une part, les épithètes homériques de gaièochos et d'ennosigaios, d'autre part, les survivances religieuses d'époque historique pour Poséidon, Déméter Malophoros et Ényalios. Calchas est d'ailleurs lié à Mégare aussi à travers la légende de son fils, Calchédon, éponyme de Chalcédoine, colonie de Mégare fondée en $684^{17}$.

$\mathrm{Si}$, de plus, le vénérable Artémision sis entre les deux acropoles de Mégare, l'archaion hiéron de Pausanias voué à la Phosphoros / Soteira, est aussi lié à la légende de fondation par Agamemnon, toute la tradition du sacrifice d'Iphigénie à Mégare dépasse de loin le cadre d'une querelle anti-athénienne. Même si le mythe d'Aulide reste canonique, grâce au témoignage de l'fliade et des Chants chypriens ${ }^{18}$, la version mégarienne constitue, avec le récit athénien, plus fameux, de Brauron, une série homogène de données culturelles typiquement mycéniennes - sur une aire géographique vaste, mais bien groupée -, dont Calchas est l'éclatant symbole.

\section{Mélampous}

Mélampous était l'objet d'un culte particulier à Égosthènes. Selon Pausanias $(I, 44,5)$, il y avait un sanctuaire avec une stèle représentant un homme de petite taille; en son honneur on accomplissait des sacrifices (thyousi) et on célébrait une cérémonie annuelle (heortè); mais les gens du lieu disaient que Mélampous ne donnait d'oracles ni en rêve ( $d i^{\prime}$ oneiraton), ni autrement. Les inscriptions confirment les données du Périégète, mentionnant un Mélampodeion ou hiéron tou Mélampodos, tout comme un concours à son nom, les Mélampodeia ou agôn tou Mélampodos ${ }^{19}$. Mélampous, qui, à Égosthènes, était un théos

15 Cf. H. BorchHARDT, Frühgriechischen Schildformen, in Kriegswesen, 1, Archaeologia Homerica, I, E, 1, Göttingen, 1977, p. E 3-5.

16 Strab., IX, 1, 10 (C394). Cf. PICCIRILl, op. cit., p. 133-134 ; RIGSBy, art. cit., p. 100-102.

17 Hsch. ML., ap. Cod. Pal. Heidelb., 398. Cf. HANELL, op. cit., p. 96.

18 Cf. PICCIRILLI, op. cit., p. 118.

$19 I G, \mathrm{VII}, 207,208,219,223$ (le sanctuaire de Mélampous était aussi l'archive de la cité). 
patrôos $^{20}$, est représenté aussi sur les monnaies de la ville de l'époque de Septime Sévère comme un enfant allaité par une chèvre 21 .

L'importance de Mélampous dans le domaine religieux mégarien est confirmée par des notices d'historiens mégariens, tel Dieuchidas, qui discutaient de son ascendance et proposaient des étiologies sur son nom $^{22}$, tout comme par un autre passage de Pausanias concernant les monuments de l'agora de Mégare : à côté de l'entrée du sanctuaire de Dionysos se trouvait le tombeau d'Astycrateia et de Mantô, filles de Polyidos, descendant de ce Mélampous qui était venu à Mégare pour purifier le roi Alcathoos du meurtre de son fils Callipolis ${ }^{23}$; Polyidos avait fondé ce temple de Dionysos (Patrôos), où il avait dédié un xoanon du dieu; une statue de Dionysos Dasyllios fut consacrée par son petit-fils Euchénor $^{24}$ (Paus., I, 43, 5).

Il n'est pas aisé de définir le caractère du Mélampous mégarien et il faut rappeler rapidement diverses hypothèses récemment présentées.

La liaison évidente entre le nom du héros - ou dieu - et l'épithète Mélanaigis de Dionysos, courante sur la triple frontière de Mégare, de l'Attique et de la Béotie (en relation aussi avec l'étymologie vraisemblable du nom d'Égosthènes) a retenu l'attention de Beschi et Musti ${ }^{25}$. Ils y décèlent un rapport évident avec les représentations monétaires de l'enfant à la chèvre, que déjà Wilamowitz et Wolff avaient rapporté à la légende de Mélampous apprenant la mantique d'un serpent qui lui aurait léché les oreilles : ainsi le devin comprenait la voix des animaux, en particulier celle des oiseaux et des insectes. Pour Wolff, en outre, on pourrait distinguer un détail important sur

20 O. WolfF, Melampus, in Roscher, Lexicon, s.v., col. 2572.

21 Ibid. Cf. J. PleY, Melampus, 1), in RE, XV, 1 (1931), col. 393 ; Hanell, op. cit., p. 102 et n. 2.

22 Mélampous, le «Pied-noir», était fils de Dorippè, selon Dieuchidas, de Rhodopè, selon une scholie à Théocrite : PICCIRILLI, op. cit., p. 42-43, 158-159.

23 Telle est notre interprétation du texte de PAUSANIAS, conformément aux éditions de N. Papachatzis, Pausaniou Hellados Periigisis, I, p. 504 et de Beschi-Musti, op. cit., p. 233. HANELL, op. cit., p. 100 et PiCCIRILli, op. cit., p. 113, comprennent différemment le passage : la venue à Mégare pour la purification d'Alcathoos est attribuée à Polyidos.

24 L'importance de telles sépultures héroïques pour la fondation des cultes civiques et l'urbanisation de Mégare a été souvent soulignée. Outre les allusions dans les ouvrages d'HANELl, PICCIRILli, BESCHI-Musti, ce sujet a donné lieu à des contributions spécifiques, telles: F. BoHRINGER, Mégare : traditions mythiques, espace sacré et naissance de la cité, in $A C, 49$ (1980), p. 5-22 ; A. Muller, Megarika VI, in BCH, 105, 1981, p. 218-222 ; BÉRARD, art. cit.

25 Op. cit., p. 439-440. 
d'autres représentations monétaires d'Égosthènes, de l'époque de Géta, où l'on voit un édifice circulaire, le Mélampodeion, caractérisé par un arbre avec un serpent enroulée 26 .

Pour Piccirilli, l'origine de Mélampous serait béotienne : puisque les Grecs appelaient les Égyptiens Mélampodes, la légende de Mélampous qui comprenait le langage des animaux - d'origine égyptienne - doit être mise en relation avec celle qui lui faisait apprendre le culte de Dionysos par Cadmos de Tyr et ses compagnons qui s'étaient rendus en Béotie. Sa légende égyptienne serait en rapport avec la Béotie, qui avait des prétentions sur la Mégaride. Les Mégariens donc, en niant les pouvoirs mantiques de Mélampous, refusaient d'une part son identification avec le devin égypto-béotien, d'autre part la tradition athénienne, qui croyait fermement dans le caractère prodigieux du héros ${ }^{27}$. Quant au tombeau d'Astycrateia et Mantô, Piccirilli pense à une invention mégarienne dépourvue de fondement ${ }^{28}$.

Partant de la théorie de Frazer que le héros est le substitut anthropomorphe d'un culte animal initial, Beschi et Musti estiment que Mélampous représente un culte local de salut, à distinguer de celui du devin argien, avec qui il se confond par la suite ${ }^{29}$. Quant à la présence de la dynastie de Mélampous dans le sanctuaire de Dionysos Patrôos et Dasyllios de l'agora de Mégare, les deux savants y voient une importation du culte dionysiaque à Mégare à partir d'Égosthènes et, audelà, de la Béotie, région particulièrement liée au dieu ${ }^{30}$.

À vrai dire, il existe déjà des incertitudes sur la figure générale de Mélampous, où s'harmonisent des éléments hétérogènes : orgiastiques (incantations, cris et danse frénétique), chthoniens (serpent, insectes, connaissance du langage animal), thaumaturgiques (médications, mélampodion = rose de Noël, pharmaka) et purificatoires (offrandes et sacrifices expiatoires et mystiques, katharmata, mantique des victimes sacrificielles) ${ }^{31}$. À telle enseigne que celui que déjà Hésiode appelait

26 WOLFF, art. cit., col. 2572.

27 PICCIRILLI, op. cit., p. 42-43.

28 Ibid., p. 113.

29 Telle était l'opinion aussi de WOLFF; PLEY, art. cit., col. 393; HANELL, op. cit., p. 104-105.

30 BESCHI-MUSTI, op. cit., p. 434-435.

31 Sur les traditions concernant Mélampous, cf. surtout WoLfF et PLEX. HANELL, op. cit., p. 99-100, le définit comme le représentant mythique des pouvoirs sacrals et prophétiques du haut archaïsme. 
"le meilleur des devins" (Schol. in A.R., I, 118) rejoint les sphères de culte d'Apollon, de Dionysos, d'Héra, d'Artémis et d'Asclépios. Il semble hors de doute, toutefois, que ses prérogatives mantiques étaient connues dès le début de la pensée grecque : il est défini par Homère comme mantis amymon (Od., XI, 291); il donne origine à une dynastie de devins célèbres à laquelle le chant XV de l'Odyssée consacre une importante digression (225-255). Cette dynastie comprenait Polyidos, devin et thaumaturge fameux. D'autres versions de sa légende le relient à Mégare, où il serait venu de Crète, et à sa colonie de Byzance, où Polyidos et ses fils étaient l'objet d'un culte et de sacrifices. Selon K. Hanell ${ }^{32}$, il est l'exemple typique de ces devins semi-mythiques du haut archaïsme, véritables prophètes itinérants.

Adraste et son fils Aigialeus, qui avaient leurs hérôa respectivement sur l'agora de Mégare et à Pagai (Paus., I, 43, 1; 44, 4; IX, 19, 2), appartenaient également à la lignée commune de Mélampous et de son frère Bias. Quant au devin peut-être le plus célèbre de cette famille (Paus., II, 23, 2), Amphiaraos, son hérôon se trouvait à Oropos, dans une région toujours contestée entre Béotie et Attique, théâtre d'importants rites de passage, tout comme le Mélampodeion était le sanctuaire le plus important d'Égosthènes, ville mégarienne toujours convoitée par les Béotiens et très sensible à leur influence politique et religieuse ${ }^{33}$. Il est évident que la proximité d'Égosthènes et du Cithéron peut expliquer le rôle joué par la lignée de Mélampous dans la diffusion du culte de Dionysos, mais la multiplicité des liens instaurés entre cette lignée et des sites différents de la Mégaride (Mégare, Égosthènes, Pagai) dépasse le cadre de la vénération pour un dieu local de salut, dont il n'y a aucune preuve. Il n'y a pas de raisons non plus d'écarter le témoignage de Pausanias, tel que nous l'interprétons (cf. supra): l'arrivée de Mélampous à Mégare est due à la purification d'Alcathoos pour le meurtre de son fils Callipolis; ce type de purification est tout à fait analogue à celle qu'opère Mélampous sur les Proïtides ${ }^{34}$.

Il n'est pas question de dresser ici un stemma de la lignée de Mélampous. De fait il y en a plusieurs, au moins trois reconnus depuis

32 HANELL, op. cit., p. 100-101.

33 Sur Egosthènes et son histoire, cf. PAPACHATZIS, op. cit., p. 511-515 et L. ROBERT, Inscriptions de Pagai en Mégaride relatives à un arbitrage, in $R P h$ (1939), p. 97122.

34 Cf. en particulier F. VIAN, Mélampous et les Proïtides, in REA, 67 (1965), p. 2530 et M. JosT, Sanctuaires et cultes d'Arcadie, Paris, 1985, p. 421-425. 
longtemps ${ }^{35}$, sans même faire référence à des travaux plus récents ${ }^{36}$. Ils se caractérisent par la présence de personnages qui portent des noms dérivés de mantis : Mantios (fils du héros) et les deux Mantô. Il y a, en effet, clairement un doublet avec une Mantô fille de Polyidos (celle qui nous intéresse pour Mégare et dont c'est ici la seule mention dans le mythe) et une Mantô fille de Tirésias et mère de Mopsos, dont la personnalité est beaucoup plus complexe : consacrée à l'Apollon de Delphes, elle fonde ensuite Claros; épouse d'Alcméon, elle est mère d'Amphilochos. Le problème est compliqué du fait que Diodore (IV, 68, 5) connaît une Mantô, fille de Mélampous, qu'on a cherché à identifier avec la nôtre ${ }^{37}$.

Les liens de Mégare avec la famille de Mélampous apparaissent en pleine lumière grâce à la version de Pausanias :

- le devin vient en personne à Mégare pour la purification du roi Alcathoos, selon l'interprétation qui nous paraît la plus vraisemblable;

- le tombeau des Mélampodides Astycrateia et Mantô jouxte le sanctuaire de Dionysos fondé par Alcathoos.

Signifiants aussi sont les liens de ces Mélampodides avec Dionysos. Le tombeau des deux filles de Polyidos est à côté du temple de Dionysos Patrôos dont Polyidos est le fondateur et où il dédie un xoanon du dieu. Une autre statue du dieu sous l'épithète de Dasyllios est consacrée par son fils Euchénor: étrange épiclèse qui ferait de lui «le dieu aux

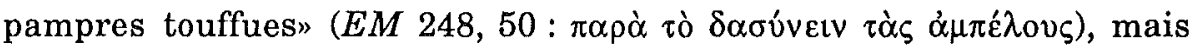
qu'il vaut mieux interpréter, selon nous, comme «à la barbe drue».

Au total Mélampous est bien à sa place dans plusieurs secteurs du monde grec et ce serait jouer d'une hypercritique funeste que de rejeter tous les témoignages qui le concernent en Mégaride, au motif qu'ils seraient des fictions locales.

Trois exégèses s'imposent à nous :

1. À Égosthènes Mélampous est un enfant divin avec des traits bien connus ailleurs : la chèvre nourricière, l'apprentissage du métier (de devin), le rôle du serpent, l'importance de l'oreille pour le mantis qui «entend» autant qu'il «voit». Certains de ces traits se trouvent, par exemple, dans le mythe de Zeus enfant, ce qui est conforme à l'hypothèse qu'il existe normalement deux registres mythiques parallèles, l'un

35 E. BERNERT, Polyidos, 1) - 3), in RE, XXI, 2 (1958), col. 1647-1658.

36 On se reportera récemment à PICcIRILLI, op. cit., p. 112-113.

37 S. EITREM, Manto, 3), in RE, XIV, 1 (1928), col. 1359 (suivant GRUPPE). 
divin, l'autre héroïque. Nous ne nous étonnons pas non plus que Mélampous soit à la fois divine child et devin adulte : Zeus cumule bien sa petite enfance crétoise avec ses destinées de dieu Père tonnant.

2. Mélampous et son lignage sont très intégrés au culte de Dionysos. Les localisations de ces épisodes ne sont pas innocentes: les témoignages concernent principalement les régions frontalières (comme, en Arcadie, un Mélampodeion est attesté aux limites d'Orchomène et de Méthydrion ${ }^{38}$ ). Par là, le héros peut aisément se révéler vecteur d'influences religieuses puissantes et conforter la diffusion du culte de Dionysos en provenance de Béotie.

3. Les personnages cités enrichissent une famille exceptionnelle, où figurent la majeure partie des grands devins grecs, trois sur cinq dans l'énumération de Cicéron ( $L g$, II, 33): Mélampous, Mopsos, Amphiaraeus, Calchas, Hélénos. C'est la race de Mélampous qui peuple aussi l'imaginaire mythique d'autres régions de l'Isthme et du Péloponnèse septentrional (Sicyone notamment). Ce qui permet de fixer un cadre chronologique précis qui est celui de la dorisation de l'Isthme par Argos aux alentours du Xe siècle.

Il reste à expliquer l'absence de mantique au sanctuaire de Mélampous à Égosthènes à l'époque de Pausanias, qui peut tenir à des impondérables que nous ignorons. Qu'on nous permette une hypothèse. Le détail de l'oniromancie auquel Pausanias fait allusion pourrait acheminer vers une solution ${ }^{39}$. S'il y a là un lien avec l'incubation et les rêves guérisseurs, la référence à la thérapeutique des temples d'Asclépios s'impose ${ }^{40}$. Au reste, l'iconographie des monnaies de Mélampous à la chèvre à Égosthènes rappelle de près certaines émissions d'Épidaure ${ }^{41}$, tout comme le bâtiment circulaire avec l'arbre et le serpent renvoie à la tholos de ce grand sanctuaire. Dans ce cas, l'absence d'oniromancie à Égosthènes trouve une explication évidente dans la spécificité d'Épidaure par rapport aux sanctuaires similaires, tels celui de Mélampous en Mégaride ou de Trophonios à Lébadée.

38 JosT, op. cit., p. 119.

39 HANELL y voit peut-être à bon droit une opposition cherchée par PAUSANIAS entre l'absence de mantique au Mélampodeion et la mantique de l'Amphiareion : op. cit., p. 104, n. 2.

40 Cf. A. TAFFIN, On rêvait dans les temples d'Esculape, in BAGB (1960), p. 325366.

41 WoLFF, art. cit., col. 2572. 


\section{L'oracle de Nuit}

L'existence à Mégare d'un oracle de Nuit est un de ces témoignages uniques - si précieux - de la Périégèse, qui ramène en plein cœur de la cité, sur l'acropole orientale, la Caria, appelée ainsi d'après le nom du roi mythique Car, fils de Phoronée. La citation de cet oracle, faite en passant par Pausanias, n'est corroborée par aucun autre indice et on ne saurait rien dire à ce propos, sauf à faire une analyse - même rapide de tout l'ensemble religieux de la Caria. Pausanias cite la présence des sanctuaires suivants: temple de Dionysos Nyktélios, hiéron d'Aphrodite Epistrophia, épimanteion de Nuit, temple de Zeus Konios, mégaron de Déméter, bâti par le roi Car, et statues d'Asclépios et Hygie par Bryaxis (I, 40,6). À part ces deux statues, qui ne peuvent dépasser le IVe siècle, l'ensemble présente un élément sûrement très ancien (voire mycénien), le mégaron de Déméter, et une certaine homogénéité dans les trois premières composantes. L'épithète Nyktélios de Dionysos renvoie aux Nyktélia, des orgies nocturnes typiquement mégariennes en relation avec le rite mystérique de la mort et de la résurrection de Dionysos, semblable à celui d'Osiris ${ }^{42}$. L'Aphrodite Epistrophia est la déesse "qui incite les hommes à l'amour" et l'on a voulu mettre en rapport l'oracle de Nuit, qui «interprète les signes de la nuit», avec la nature chthonienne de cette Aphrodite ${ }^{43}$. L'épiclèse locale de Zeus, Konios, "Poussiéreux», demeure obscure : elle est peut-être en relation avec le pouvoir des vents de soulever la poussière ${ }^{44}$. Dans ce cas on ne sortirait pas du caractère chthonien commun à toutes ces divinités.

L'énigme la plus complète demeure au sujet de l'oracle de Nuit, vu l'absence totale de comparaison. Nulle part, il n'est explicitement question de sanctuaires ou d'autels érigés en l'honneur de Nuit. À part le cas de Mégare, Nuit est mise en cause pour l'oracle de Delphes par les scholies aux Pythiques de Pindare (Schol. in Pind., P., hypothesis, a): elle aurait détenu le manteion avant Thémis, Python, Dionysos et Apollon. Dans un autre texte d'allure orphique très accentuée (Plut., $D e$ se. num. vin., $28=$ Moralia, $566 \mathrm{~b}-\mathrm{c}$ ), Plutarque affirme qu'il n'y a rien

42 BESCHI-MUSTI, op. cit., p. 423.

43 Ibid., p. 423-424.

44 Ibid., p. 424. PAPACHATZIs, op. cit., p. 496, n. 1, offre d'autres explications de l'étrange épiclèse : Konios pourrait avoir le sens de «donneur de gloire», connexe à la poussière qui se lève dans le champ de bataille, ou être en relation avec l'argile des gymnases. Cf. P. Chantraine, Dictionnaire étymologique de la langue grecque, s.v. : «qui soulève la poussière». 
de commun entre Apollon et Nuit. Celle-ci aurait, en revanche, un manteion avec Sélénè qui ne serait pas borné à un emplacement précis de la terre, mais flotterait avec les rêves et les phantasmes des hommes. Dans les cosmogonies orphiques, en effet, Nuit représente une entité originale, qui prend la place d'Océanos et Téthys. Déjà dans Homère, un passage de l'Iliade (XIV, 258-261) semble conférer à Nyx l'autorité et le pouvoir que Zeus, bien que souverain, doit reconnaitre à une puissance primordiale ${ }^{45}$. Mais c'est avec Hésiode que Nuit conquiert une place canonique dans la cosmogonie grecque : Nuit est, avec Érèbe, fille de Chaos; des ouvres de son frère, elle enfante Éther et Jour (Hêmerê) (Th., 123-125). «Dans ce groupe de quatre, ... Érebos est à Nyx ce que Aither est à Hêmerêm. Si Jour et Nuit ne sont pas dissociables, Éther et Érèbe correspondent en revanche aux formes extrêmes et exclusives d'un Blanc et d'un Noir sans partage ${ }^{46}$. Par la suite, au terme du processus cosmogonique, on voit réapparaître les enfants de Nuit, quand un nouvel ordre va s'instaurer, avec la séparation d'Ouranos de Terre par l'acte de violence de Cronos. Il est signifiant que toutes les forces d'obscurité que Nuit enfante sans s'unir à quiconque sont contemporaines des Gégéneis, nés de Gaia par les éclaboussures d'Ouranos, et d'Aphrodite. Aux «fils de Terre» correspondent les "fils de Nuit» : aux Érinyes s'opposent Némésis et les Kères; aux Géants et aux Nymphes des frênes, Lutte (Eris stugerê) avec son cortège de Mêlées, Combats, Meurtres et Tueries; à Aphrodite, caractérisée par les babils des jeunes filles, les tromperies et l'Union amoureuse, s'opposent Mots menteurs (Pseudea), Tromperie (Apatê), Union amoureuse (Philotês) (Th., 211-232) $)^{47}$.

À la lumière de ces remarques, il est hautement significatif qu'à Mégare l'oracle de Nuit accompagne Dionysos Nyktélios et Aphrodite Epistrophia. Après la séparation de Ciel et de Terre, ce sont les divinités des passages chthoniens, Dionysos par excellence, qui assurent la communication entre le bas et le haut, le visible et l'invisible, la vie et la mort. Aphrodite aussi acquiert, dans cette perspective, un rôle singulier, car on peut entrevoir un double caractère de la puissance amoureuse, bénéfique et trompeuse, puisqu'il y a aussi l'Union menteuse parmi les Fils de Nuit. De plus, la violence sexuelle est à l'origine des Cronides aussi bien que de la séparation entre Jour et Nuit : la naissance de la

45 J.-P. Vernant, Cosmogoniques (mythes). La Grèce, in Dictionnaire des mythologies, sous la dir. de Y. BONNEFOY, Paris, 1981, p. 254.

46 Ibid., p. 256.

47 Ibid., p. 260. 
déesse de l'amour, synchrone des enfantements solitaires de Nuit, en est l'extrême conséquence.

La présence du mégaron de Déméter sur la Caria ne s'oppose pas à ce tableau «nocturne», surtout s'il faut reconnaître dans la caverne de Mourmouni, qui s'ouvre sur les pentes de l'acropole, la Pétra Anaklèthra nommée par Pausanias, la falaise de laquelle Déméter appelait sa fille Korè, lorsqu'elle errait à sa recherche (Paus., I, 43, 2). Selon l'identification très convaincante de A. Muller, il s'agirait d'une «entrée des Enfers", où Déméter vint chercher sa fille. "Les Mégariennes célébraient encore, du temps de Pausanias, une fête annuelle, au cours de laquelle elles mimaient la quête de la déesse et son appel devant l'entrée des Enfers : il est probable que la petite terrasse aménagée devant l'entrée de l'antre de Mourmouni servait de scène à ce drame sacré $48^{\text {». }}$

Au cosmos nocturne et chthonien de la Caria s'opposait, à Mégare, «la lumière poliade» de l'autre acropole, l'Alcathoos, marquée par les signes d'un véritable rituel de fondation civique. Pour reprendre les

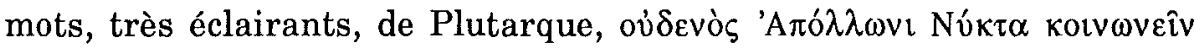
(Plut., ibid.). L'opposition structurale entre Nuit et Apollon est celle qui rend fonctionnelle, à Mégare, avec le partage des deux acropoles, l'insertion des puissances primordiales et pré-olympiennes dans le panthéon poliade. L'action d'Apollon se déploie, dans toute sa puissance démiurgique, dans le mythe de fondation de l'acropole même, qui l'associe, dans la construction du mur d'enceinte, à Alcathoos, oikistès divin à côté de l'oikistès humain ${ }^{49}$. On vénérait même le rocher sur lequel Apollon avait posé sa lyre, pour mettre la main à l'édification du mur (Paus., I, 42, 2). Cette fondation était marquée par un sacrifice sur le foyer (hestia) des dieux Prodomeis (Paus., I, 42, 1). Au sommet de l'acropole trônaient les temples des dieux poliades par excellence, Athéna (Nikè et Aiantis) (Paus., I, 42,4) et Apollon, sous les épiclèses de Pythios, Dékatéphoros et Archégète (Paus., I, 42,5). Divinité sans aucun doute la plus importante du panthéon local, l'Apollon de Mégare ne

48 A. Muller, Megarika II, in BCH, 104 (1980), p. 91. Cf. ID., Megarika I, ibid., p. 83-89, pour la réfutation de l'attribution courante de la caverne de Mourmouni au mégaron de Déméter.

49 Le mythe d'Alcathoos, lié à l'acropole occidentale et aux dieux poliades, est le plus étudié, parmi les traditions mégariennes : cf. PICCIRILLI, op. cit., p. 38-41, 108-109; Bohringer, art. cit., passim; Muller, De Nisée à Mégare, p. 623-628; BÉRARD, art. cit., p. 636-639. 
semble avoir eu aucun pouvoir mantique, même pas dans sa manifestation rurale de Tripodiscos, qui, comme le nom et le mythe connexe le montrent, renvoyait au symbole apollinien du trépied delphique ${ }^{50}$. Cet épisode même dénote une convergence entre l'Apollon Pythien d'Argos et celui de Delphes dans un pays comme la Mégaride, exposé à ces deux influences. Le caractère poliade de l'Apollon de l'Alcathoos de Mégare s'accorde bien avec les traditions sur la dorisation du pays, d'origine argienne.

À travers cette organisation politique et civique précise qui aboutira au synœcisme dès la fin du VIIIe s., consacrée par l'image d'un Apollon oikistès et "dépositaire de la lumière», les manifestations des puissances mantiques et oraculaires locales, toutes antérieures à la genèse de la polis et expression d'un cosmos différent, survivent aux marges du pays, ou aussi en son cœur même, sous la protection de Nuit "qui dompte hommes et dieux» (Il., XIV, 259).

Au total, la Mégaride offre, sur le thème de la mantique, une documentation modeste, mais doublement signifiante.

D'une part, s'imposent les personnages de Calchas et de Mélampous, qui attirent l'attention sur une vigoureuse strate mycénienne et géométrique, dans une large problématique : liaison du pouvoir avec ces voyants itinérants qui jouent aussi un rôle dans la diffusion des influences religieuses, charge sacrée des frontières où se rencontrent des cultures spécifiques, homologies avec les zones voisines qui emmagasinent et exploitent le même potentiel mythique...

D'autre part, le très étonnant oracle de Nuit permet peut-être une meilleure compréhension des deux acropoles de Mégare et de leurs significations complémentaires, l'une pleinement vouée aux cultes anciens, mycéniens, l'autre en liaison étroite avec la fondation de la cité sous le patronage d'Apollon.

Claudia ANTONETTI et Pierre LÉVÊQUE

Istituto italiano per la storia romana

et

Université de Besançon

Faculté des Lettres et Sciences humaines

50 Sur la tradition mythique de Tripodiscos, cf. PICCIRILLI, op. cit., p. 122-125. Sur les possibilités d'un caractère mantique de l'Apollon Pythien de Mégare, $\mathrm{cf}$. HANELL, op. cit., p. 87, 89 ; ibid., p. 172, pour l'oracle d'Apollon Chréstérios de Chalcédoine. Pour la négation du caractère mantique de l'Apollon de Mégare, cf. RIGSBY, art. cit., p. 415. 